\title{
CONFERENCIAS
}

\section{ALTERACIONES ELECTROLITICAS Y DEL METABOLISMO DEL AGUA EN LOS PROCESOS NEUROLóGICOS}

\author{
MaRIo Michel ZaMora *
}

El concepto del control de las funciones metabólicas por centros situados en el cerebro fué ya enunciado por Claude Bernard, en 1849, cuando dió a conocer que la punción del cuarto ventrículo determinaba glicosuria. Dentro de estas alteraciones producidas por lesiones cerebrales se encuentra la diabetes insípida. Fisher y col. han demostrado que la interrupción de más del $50 \%$ de las fibras del haz supraóptico-hipofisario en el hipotálamo del gato desencadenaba diabetes insípida. Asímismo, la sección del infundibulo suficientemente alta como para desprender la mayor parte de la eminencia media de la base del hipotálamo, o la extirpación de la neurohipófisis incluyendo la eminencia media, producía invariablemente y en forma permanente la diabetes insípida. De los estudios citados, se llega a la conclusión de que la diabetes insipida es el resultado de la producción deficiente del principio antidiurético en la neurohipófisis. Según la teoría de la neurosecreción de Scharrer, son las células de los núcleos supraóptico y otros similares del hipotálamo las que segregan una substancia relacionada con la hormona antidiurética la que luego es transportada a lo largo de los axones al lóbulo posterior de la hipófisis, el que actuaría como depósito dando salida a dicha substancia hacia el sistema vascular de la pituitaria por estímulos transmitidos a través de los cilindroejes del haz supraóptico-hipofisario. Dichos estímulos son producidos por alteraciones en la osmolaridad, composición y volumen del líquido extracelular, así como por estímulos psíquicos y por ciertas substancias químicas. El tratamiento sintomático de la diabetes insípida se hace a la base de extractos de lóbulo posterior de hipófisis. Court y Taylor han propuesto el empleo de preparaciones de tanato de pitresin con aceite, como un medicamento que sería de acción antidiurética lenta.

Los electrólitos y principalmente el potasio tienen una acción importante en la actividad funcional nerviosa, ya que sus cambios van ligados a modificaciones de la permeabilidad de la membrana celular y a desviaciones

* Profesor de Neurología y Neurocirugia de la Facultad de Medicina de La Paz, Bolivia. 
físico-químicas de su potencial eléctrico. Durante la estimulación de nervios se libera potasio (Hober, Cowan, Young) y también la estimulación eléctrica del cerebro origina aumento del potasio en la sangre del seno longitudinal superior. En la preparación del "encéfalo aislado" del gato y con inyecciones intracarotídeas de potasio observan Bonnet y Bremer que pequeñas dósis $(0,1$ a $0,5 \mathrm{mgr}$.) actúan estimulando las células corticales, con aumento de la actividad eléctrica de la corteza; después de esta fase de excitación aparece un período de depresión; dósis más elevadas suelen producir solamente depresión que puede llegar a la extinción completa.

En enfermos epilépticos han sido estudiadas la conducta y variaciones de los electrólitos. Penfield y Erickson afirman que en los períodos interparoxísticos los distintos componentes sanguíneos del metabolismo iónico (calcio, fósforo, potasio, magnesio, cloro y sodio) son normales y que después de los ataques aumentan secundariamente y como consecuencia de los mismos el calcio y el fósforo. Otros autores han observado que la aparición de convulsiones va precedida de un aumento del potasio sanguíneo y de su eliminación y Mc. Quarrie deduce que existe una pérdida de potasio de las células debido a un aumento de permeabilidad de la superficie celular, lo que considera como factor etiológico fundamental en la génesis de la descarga epiléptica. Un estudio del metabolismo de los epilépticos durante períodos de control y tratamiento ha demostrado también (Cohen, Coombs, Cobb y Talbott) que en los períodos sin convulsiones la eliminación de potasio era baja y que en los períodos con ataques frecuentes aumentaba la eliminación de potasio; los autores citados no pueden definir si la retención del potasio es un efecto coincidente o causal con la acción anticonvulsivante.

En lo que respecta al metabolismo del agua en los epilépticos, se sabe que se pueden desencadenar ataques en un $40 \%$ de los casos mediante la ingestión continuada, durante varias horas, de gran contidad de agua, favoreciendo su retención con inyecciones repetidas de hormonas del lóbulo posterior de la hipófisis que inhiben la diuresis. Durante esta prueba de hidratación, se han visto marcadas alteraciones en el electroencefalograma. La hidratación e intoxicación acuosa en ratas producía una disminución del umbral convulsivante para estímulos químicos y eléctricos y aparecían ondas lentas y potenciales similares a los de los enfermos epilépticos. Supone Mc. Quarrie que el agua pasa a las células nerviosas y que éstas pierden potasio. En la práctica, se han recomendado curas de deshidratación en enfermos epilépticos pero su empleo no ha llegado a alcanzar mucha difusión.

El equilibrio ácido-base del organismo tiene tämbién gran importancia. La alcalosis precipita los ataques y la acidosis los inhibe. Esta afirmación se halla confirmada en la práctica con el empleo de la dieta cetógena, introlucida por Wilder en 1921 y con la cual muchos pacientes, especialmente niños con Petit Mal, experimentan una notable mejoría. También se ha comprobado que la dieta indicada disminuye la actividad anormal del electroencefalogram en niños con epilepsia. Se piensa que el modo de actuar de la dieta cetógena es por la acidosis y deshidratación que determina (el Diamox actúa también en esta forma). 
Nyan y Cooke han presentado casos de hiponatremia secundaria a infecciones agudas del sistema nervioso central. Sus observaciones se refieren a cinco niños que tenían meningitis causada por diferentes gérmenes patógenos y en los que se observó signos y síntomas de intoxicación por el agua: excesiva inquietud o desasosiego, irritabilidad exagerada o convulsiones con marcada disminución del sodio en el suero, cuya tasa varió entre 112 y $125 \mathrm{mEq} / \mathrm{l}$; el cloro de 86 a $94 \mathrm{mEq} / 1$ y el potasio de 22 a $4.2 \mathrm{mEq} / \mathrm{l}$. Se obtuvo una dramática mejoría en estos pacientes al corregir la hiponatremia mediante la administración de cloruro de sodio. Una descarga excesiva de hormona antidiurética por parte del lóbulo posterior de la hipófisis parece ser la causa de la limitada capacidad de eliminar agua en estos enfermos. Los autores creen que la descarga de hormona antidiurética sea resultado de la estimulación del núcleo supraóptico por la reacción inflamatoria aguda del sistema nervioso central. Como conclusión y desde el punto de vista práctico, debe limitarse en forma cuidadosa la ingestión y administración de agua en las infecciones agudas del sistema nervioso. Los enfermos que requieran líquidos por la vía parenteral reciben inicialmente 10-15 ml/kg de solución de lactato de Ringer, o de Hartmann. Esta medicación se la efectúa incluso en caso de que no haya evidencia clínica de deshidratación ya que puede existir cierta depleción del volumen extracelular. Posteriormente la administración de líquido se da en una proporción de 60 a $80 \mathrm{ml} / 100$ calorías metabolizables de una solución que contenga $3 \mathrm{mEq}$ de sodio y $2 \mathrm{mEq}$ de potasio en dicho volumen. En esta forma se evita la hiponatremia. Igual regla debe seguirse en la administración de líquidos por la vía oral ya que el mecanismo de la sed puede hallarse alterado. Posteriormente, se deberá efectuar determinaciones de electrólitos y nitrógeno no proteico para continuar con la atención del paciente.

También se observa hiponatremia en la meningitis tuberculosa, sin sintomas o muy escasos referibles a la baja concentración de disolvente. Asímismo, varios autores han señalado que en otro tipo de hiponatremia la pérdida de sodio se acompaña de deshidratación, hipotensión y azotemia y se presenta en diferentes procesos cerebrales como encefalitis, poliomielitis bulbar, accidentes cerebrovasculares y tumores del cuarto ventrículo.

Es interesante hacer notar que otra anormalidad de los electrólitos en procesos cerebrales es la marcada retención de sodio y cloro en el plasma. El primer trabajo sobre este transtorno fué publicado en 1939 por Allot presentando cinco casos en los cuales dicha anormalidad bioquímica fué notada en el curso de afecciones cerebrales de curso fatal. Estos pacientes presentaron hipernatremia, hipercloremia y uremia asociadas a una marcada disminución de la excreción urinaria de sodio y cloro. Sweet y asociados han publicado el caso de una enferma de 47 años de edad en la que se practicó una lobotomía y que murió a los 32 dias después de la operación y que presentó hipernatremia, hipercloremia, hiperglicemia, uremia y hemorragias gastrointestinales. Biemond ha descrito un sindrome de oliguria inter- 
mitente con edema de la cara e hipernatremia sccundarias a una lesión hipotalámica en una mujer de 32 años. Cooper da cuenta de siete casos con lesiones en la hipófisis, craneofaringeoma, accidente cerebrovascular de los núcleos de la base, tumor de tercer ventrículo, higroma subdural y trastorno del desarrollo cerebral en los que registró altas cifras de sodio del suero (de 155 a $170 \mathrm{mEq}$ ) y de cloruros del plasma (entre 127 y $134 \mathrm{mEq} / 1$ ). Este mismo autor, en una revisión de 3.000 casos de pacientes, encontró 9 en los que el sodio en sangre se hallaba elevado a $155 \mathrm{mEq} / \mathrm{l}$, o más.

Los hallazgos idénticos de anormalidades electrólitas por diferentes investigadores en lesiones cerebrales localizadas en el lóbulo frontal o en el hipotálamo, permiten afirmar que dichas alteraciones son secundarias a Ia lesión cerebral, lo que también se halla apoyado por la investigación fisiológica. Lewy y Gasman han dado a conocer altos niveles de cloruro en la sangre de gatos después de lesionar el núcleo paraóptico del hipotálamo el que se halla funcionalmente relacionado con la porción nerviosa de la hipófisis. La importancia práctica de estas anormalidades en los electrólitos son bien claras. Cooper piensa que la alta mortalidad de las operaciones en la región del tercer ventrículo y en el hipotálamo atribuída a lesión de "centros vitales", sin que se haya dado una causa precisa, puede deberse a dichas alteraciones bioquímicas.

En la parálisis periódica hay una disminución del potasio del suero y los transtornos musculares se hallan en relación con la migración de dicho ion del suero a los espacios extramusculares, posiblemente a los espacios intracelulares. La desaparición de la parálisis se acompaña de niveles normales de potasio en el suero.

De acuerdo a estudios metabólicos efectuados después de una craneotomia, se sabe que las alteraciones halladas son semejantes a las observadas después de otras intervenciones quirúrgicas: hay retención de sodio, pérdida de potasio y de nitrógeno y frecuentemente oliguria. Wise cita dos casos en los que la hiponatremia después de craneotomía por tumores de la pituitaria, hizo pensar en la existencia de hematomas intracraneales habiendo sido reoperado uno de los pacientes con resultado negativo; la mejoría rápida del estado de inconsciencia en que se hallaban mediante la inyección de solución salina hipertónica fué dramática. Respecto a la administración de líquido después de una craneotomía, no se puede dar una figura arbitraria pues hay varios factores variables tales como clima, peso del cuerpo y temperatura que influyen en la pérdida de liquidos. Si se administran soluciones salinas la cantidad no requiere un cálculo exacto y sólo debe tenerse en cuenta el estado cardíaco del enfermo y la cantidad de agua que se administra. Si se emplean soluciones libres de electrólitos, el volumen debe calcularse de acuerdo a la eliminación de orina y las pérdidas de agua por otras vías incluyendo la extrarrenal. Como ya se ha indicado, puede haber oliguria después de una craneotomía y persistir por varios días. Una eliminación de orina de 500 a 700 mililitros puede considerarse adecuada los primeros días después de una operación seria. Si se trata de aumentar dicho volume administrando líquidos durante ese tiempo, se produce reten- 
ción, que puede averiguarse fácilmente pesando al enfermo, o en otras palabras, un enfermo debe perder peso después de una operación y si esto no ocurre es por que hay retención de líquidos.

Finalmente, debemos llamar la atención sobre la pérdida de nitrógeno que se produce después de operaciones en el cerebro, transtorno que se acentúa en las lesiones traumáticas de la médula espinal. La pérdida de nitrógeno y la hipoproteinemia que es su consecuencia, es de fácil observación en el paciente paraplégico y es una de las causas de la úlcera de decúbito que hace tan dificil su atención. Es por este motivo que desde hace ya algún tiempo administramos a estos enfermos testosterona y últimamente uno de sus derivados, el fenilpropionato de 19-nor-andrestenolona (Durabolin), que tiene un mayor efecto anabolizante $\mathrm{y}$ menor acción virilizante.

De todo lo expuesto, se ve la importancia que tiene el conocimiento de las alteraciones electrolíticas y del metabolismo del agua en Neurología y Neurocirugía, y tal vez no esté lejano el día en que estos cambios bioquímicos sean más exactamente conocidos, para um mejor cuidado del enfermo.

\section{REFERENCIAS}

1. COOPER, I. S.; MAC CARTHY, C. S. - Unusual electrolyte abnormalities associated with cerebral lesions. Proc. Staff Meet. Mayo Clin., 26:354, 1951. 2. MAC CARTY, C. S.; COOPER, I. S. - Neurologic and metabolic effects of bilateral ligation of the anterior cerebral arteries in man. Proc. Staff Meet. Mayo Clin., 26:185, 1951. 3. COOPER, I. S. - Disorders of electrolyte metabolism in diseases of the central nervous system. Neurology, 3:119, 1953. 4. WISE, B. L. - Fluid and electrolyte balance following craniotomy. J. Neurosurg., 13:223, 1956. 5. NYHAN, W. L.; COOKE, R. E. - Symptomatic hyponatremia in acute infections of the central nervous system. Pediatrics, 18:604, 1956. 6. WILT, L. G.; SELDING, D. W.; NELSON, W. P.; GERMAN, W. J.; PETERS, J. P. - Role of the central nervous system in metabolism of electrolytes and water. A.M.A. Arch. Int. Med., 90:355, 1952.7. PORTER, H. - Amino acid excretion in degenerative diseases of the nervous system. J. Lab. a. Clin. Medicine, 34:1623, 1949. 